\title{
SOLUTIONS OF PERIOD THREE FOR A NON-LINEAR DIFFERENCE EQUATION
}

\author{
A. BROWN'
}

(Received 2 May 1983)

\begin{abstract}
The paper uses the factorisation method to discuss solutions of period three for the difference equation$$
x_{n+1}=a x_{n}^{3}+(1-a) x_{n} \quad(0<a \leqslant 4) \text {, }
$$

which has been proposed as a simple mathematical model for the effect of frequency dependent selection in genetics. Numerical values are obtained for the critical values of $a$ at which solutions of period three first appear. In addition, the interval in which stable solutions are possible has been determined. Exact solutions are given for the case $a=4$ and these have been used to check the results.
\end{abstract}

\section{Introduction}

One of the problems in discussing the evolution of plants and animals is to explain the persistence of large amounts of genetic variation in populations. Rarer characteristics do not always remain rare, or die out, and in a number of instances rarer characteristics appear to be favoured, so that their frequency increases from one generation to the next. This can arise, for instance, in mating patterns where males with rare characteristics are given preference by the females or in a predator-prey situation where the predator tends to select prey which show the more common characteristics. Clarke $[3,4]$ has reviewed the evidence for frequency-dependent selection effects and concludes that they are a powerful,

\footnotetext{
'Department of Theoretical Physics, Research School of Physical Sciences, Australian National University, G.P.O. Box 4, Canberra, A.C.T. 2601.

(1) Copyright Australian Mathematical Society 1984, Serial-fee code 0334-2700/84
} 
perhaps a dominant, factor in maintaining genetic diversity. Briefer references to frequency-dependent selection appear in Parkin [10], Falconer [5] and Lewontin [7].

If we consider populations with discrete generations and look for a mathematical model of frequency-dependent selection, we can write $p_{n}$ for the frequency in the $n$th generation, with $0 \leqslant p_{n} \leqslant 1$, and take $p_{n+1}$ as a function of $p_{n}$. For the effect mentioned above we want to have $p_{n+1}>p_{n}$ when $p_{n}$ is close to zero and $p_{n+1}<p_{n}$ when $p_{n}$ is close to 1 . To provide a simple model, May [8] suggested that the equation

$$
x_{n+1}=F\left(x_{n}\right)=a x_{n}^{3}+(1-a) x_{n}
$$

merited investigation, where $x_{n}=2 p_{n}-1$, with $-1 \leqslant x_{n} \leqslant 1$. If the parameter $a$ is positive, then $x_{n+1}>x_{n}$ (and $p_{n+1}>p_{n}$ ) for $-1<x_{n}<0$, with $x_{n+1}<x_{n}$ and $p_{n+1}<p_{n}$ for $0<x_{n}<1$. This agrees qualitatively with the idea that the rarer genotypes are favoured and it offers some mathematical simplification in that

$$
F\left(-x_{n}\right)=-F\left(x_{n}\right) \text {. }
$$

For $a>1, F\left(x_{n}\right)$ gives two turning values in the mapping from $x_{n}$ to $x_{n+1}$ and in this respect it goes beyond the "one hump" mappings that have mainly been considered in population growth problems. To agree with the genetics problem we must have $a \leqslant 4$, since this ensures that $\left|x_{n+1}\right| \leqslant 1$ when $\left|x_{n}\right| \leqslant 1$, so it will be assumed throughout that $0<a \leqslant 4$.

In the paper cited above [8] and in subsequent work [9], May showed that solutions with period 2 are available for $a>2$ and he discussed these solutions in detail. The present paper deals with the corresponding problem for solutions with period 3. It establishes the critical values for their appearance and the intervals in which these solutions are stable. This is done by using the factorisation method which was introduced in considering the logistic difference equation $[1,2]$.

In Section 2, some general properties of equation (1.1) are considered and it is shown that exact solutions are available when $a=4$. In Section 3 , the notation and procedure of the factorisation method are introduced by applying it to solutions of period 2. Section 4 builds on this and extends it to the more complex case of solutions with period 3. In this section equations are established which allow the solutions of period 3 to be found for any value of $a$ (if such solutions exist). Numerical results from this are considered in Section 5.

\section{Some properties of the difference equation}

If we take the corresponding differential equation as

$$
d y / d t=b\left(y^{3}-y\right)
$$


with $b>0$ and $y(0)=y_{0}$, where $\left|y_{0}\right|<1$, then the solution is of the form

$$
y^{2}=1 /\{1+D \exp (2 b t)\} \text {, }
$$

where $D$ is a positive constant. As $t$ increases, $y$ tends to zero and the approach is from one side only. In this case, $y=0$ is a stable equilibrium point and there are unstable equilibrium points at $y=+1$ and $y=-1$. If we write $x_{n}=y(n h)$, with $h>0$, and replace $d y / d t$ at $t=n h$ by $(1 / h)\left(x_{n+1}-x_{n}\right)$, then we need only put $a=b h$ to get equation (1.1). The equilibrium points of the difference equation are still at 0,1 , and -1 and when $a$ is small enough the solution of the difference equation behaves in the same way as the solution of the differential equation. For $0<a \leqslant 2$, the equilibrium point at $x_{n}=0$ is stable, with $x_{n}$ tending to zero from one side only for $0<a \leqslant 1$ and oscillating about zero, ultimately, for $1<a \leqslant 2$.

If $0<x_{n}<1$, then from equation (1.1)

$$
\begin{gathered}
x_{n+1}-x_{n}=a x_{n}\left(x_{n}^{2}-1\right)<0, \\
x_{n+1}+1=a\left(x_{n}+1\right)\left\{x_{n}^{2}-x_{n}+(1 / a)\right\} .
\end{gathered}
$$

The quadratic factor in equation (2.4) is positive definite for $0<a<4$ and this means that $-1<x_{n+1}<x_{n}<1$. In the same way, it can be shown that $-1<x_{n}$ $<x_{n+1}<1$ if $-1<x_{n}<0$. Since $x_{n+1}=0$ when $x_{n}=0$, it follows that, for $0<a<4,\left|x_{n+1}\right|<1$ if $\left|x_{n}\right|<1$, so if we start with $\left|x_{0}\right|<1$ then all subsequent values of $x_{n}$ satisfy $-1<x_{n}<1$.

For $a=4$ and $\left|x_{0}\right|<1$, write $x_{0}=\cos \phi$. Then

$$
x_{1}=4 x_{0}^{3}-3 x_{0}=\cos 3 \phi
$$

and, continuing in the same way, $x_{n}=\cos \left(3^{n} \phi\right)$. Thus equation (1.1) has an exact solution for $a=4$. Note that in this case we can start with, say, $0<x_{0}<1$ and have $x_{n}=+1$ after a finite number of steps (for example, take $\phi=2 \pi / 729$ ). Thereafter $x_{n}$ would remain at the equilibrium level. In the same way, $x_{n}$ could become 0 or -1 after a finite number of steps and then remain at the equilibrium level.

Another property for $a=4$ is that we can write down the periodic solutions for any given period. For period $M$, we must have $\cos \phi=\cos \left(3^{M} \phi\right)$ and this is satisfied when $\phi=2 N \pi /\left(3^{M} \pm 1\right)$, for any integer $N$. For example, when $M=2$ there are three periodic solutions and they can be obtained by taking $\phi=\pi / 4$, $\phi=\pi / 5$ and $\phi=4 \pi / 5$. The second and third of these are "mirror images" of each other in the sense that the solution elements are $\{\cos (\pi / 5), \cos (3 \pi / 5)\}$ in one case and $\{-\cos (\pi / 5),-\cos (3 \pi / 5)\}$ in the other. For $M=3$, appropriate values of $\phi$ are $\pi / 13,2 \pi / 13, \pi / 14$ and $2 \pi / 14$. These give four solutions for which the sum of the elements is positive. For each of these there is a corresponding mirror image solution, obtained by changing the sign of each element. Thus in all there are eight distinct solutions with period 3. These exact solutions were of great value in checking some of the later results. 


\section{Solutions with period 2}

For $a>2$, solutions with period 2 can occur. If we exclude the equilibrium solutions, we want $b_{1}$ and $b_{2}$, with $b_{1} \neq b_{2}$ and $0<\left|b_{i}\right|<1$, such that

$$
\begin{aligned}
& b_{2}=F\left(b_{1}\right)=a b_{1}^{3}+(1-a) b_{1}, \\
& b_{1}=F\left(b_{2}\right)=a b_{2}^{3}+(1-a) b_{2} .
\end{aligned}
$$

If we write $\alpha=b_{1}+b_{2}$ and $\beta=b_{1} b_{2}$, then $b_{1}$ and $b_{2}$ are the roots of the equation $x^{2}-\alpha x+\beta=0$ and can be determined if we can find $\alpha$ and $\beta$. To do this, we express $\beta$ as a function of $\alpha$ and then derive an equation for $\alpha$.

From equations (3.1) and (3.2),

$$
b_{2}-b_{1}=\left(b_{1}-b_{2}\right)\left\{a\left(b_{1}^{2}+b_{1} b_{2}+b_{2}^{2}\right)+(1-a)\right\} .
$$

Cancelling a non-zero factor $b_{1}-b_{2}$, we get

$$
a-2=a\left(b_{1}^{2}+b_{1} b_{2}+b_{2}^{2}\right)=a\left(\alpha^{2}-\beta\right),
$$

or

$$
\beta=\alpha^{2}-1+(2 / a) .
$$

This gives $\beta$ as a function of $\alpha$ and adding equations (3.1) and (3.2) gives

$$
\alpha=\alpha\left\{a\left(\alpha^{2}-3 \beta\right)+(1-a)\right\} .
$$

If we use equation (3.4) to replace $\beta$, we get

$$
0=2 \alpha\left(a-3-a \alpha^{2}\right),
$$

which is our equation for $\alpha$. From this equation

$$
\text { (i) } \alpha=0 \text { or (ii) } \alpha^{2}=(a-3) / a \text {. }
$$

(i) When $\alpha=0, b_{2}=-b_{1}$ and $\beta=-b_{1}^{2}$, so equation (3.4) gives

$$
b_{1}^{2}=-\beta=1-(2 / a)=(a-2) / a \text {. }
$$

This gives a real non-zero solution for $a>2$ and we can take $b_{1}=k, b_{2}=-k$, where $k=\sqrt{(a-2) / a}$.

(ii) When $\alpha^{2}=(a-3) / a$, we get $\alpha=0$ for $a=3$ and this brings us back to case (i). For $a>3$, there are two solutions, with $\alpha= \pm \sqrt{(a-3) / a}$ and with $\beta=-1 / a$ in both cases. This is to be expected, for if $b_{1}=F\left(b_{2}\right)$ and $b_{2}=F\left(b_{1}\right)$, with $b_{2} \neq-b_{1}$, then

$$
F\left(-b_{1}\right)=-F\left(b_{1}\right)=-b_{2}, \quad F\left(-b_{2}\right)=-F\left(b_{2}\right)=-b_{1},
$$

which verifies that $\left(-b_{1},-b_{2}\right)$ also provides a solution. For this pair of mirror image solutions, $\beta$ should have the same value but $\alpha$ changes sign, which is what we have above. 
It will be observed that in the factorisation method we avoid forming $F\left\{F\left(x_{n}\right)\right\}$ explicitly and instead work with relatively simple equations for the symmetric functions $\alpha$ and $\beta$.

For these solutions with period 2 , the condition for stability is that $-1<S_{2}<1$, where

$$
\begin{aligned}
S_{2} & =F^{\prime}\left(b_{2}\right) F^{\prime}\left(b_{1}\right)=\left\{3 a b_{2}^{2}+(1-a)\right\}\left\{3 a b_{1}^{2}+(1-a)\right\} \\
& =9 a^{2} B+3 a(1-a) A+(1-a)^{2},
\end{aligned}
$$

with $B=b_{1}^{2} b_{2}^{2}=\beta^{2}$ and $A=b_{1}^{2}+b_{2}^{2}=\alpha^{2}-2 \beta$. We can write

$$
S_{2}=\{3 a \beta-(1-a)\}^{2}+3 a(1-a) \alpha^{2}
$$

and insert in turn

$$
\text { (i) } \alpha=0, \beta=(2-a) / a, \quad \text { (ii) } \alpha^{2}=(a-3) / a, \beta=-1 / a \text {. }
$$

This gives $S_{2}=(2 a-5)^{2}$ in case (i) and $S_{2}=7+4 a-2 a^{2}$ in case (ii), in agreement with the results given by May [8]. From these expressions, the solutions with $\alpha=0$ are stable for $2<a<3$ and the solutions with $\alpha \neq 0$ are stable for $3<a<1+\sqrt{5}$. Thus it is possible to have two stable cycles of period 2 and one unstable cycle for the same value of $a$.

\section{Solutions of period 3}

If we iterate equation (1.1) three times we would get $x_{n+3}$ as a polynomial of degree 27 in $x_{n}$ and equating $x_{n+3}$ to $x_{n}$ would give a polynomial equation of degree 27 for $x_{n}$. However, $x_{n+3}=x_{n}$ is satisfied by the equilibrium solutions $x_{n}=0,1,-1$, so we can expect to have $x_{n}\left(x_{n}^{2}-1\right)$ as a factor, leaving a polynomial equation of degree 24, say $G(x)=0$. A particular solution of period 3, with elements $b_{1}, b_{2}, b_{3}$ will contribute a factor $h(x)$ to $G(x)$, where

$$
h(x)=\left(x-b_{1}\right)\left(x-b_{2}\right)\left(x-b_{3}\right)=x^{3}-\alpha x^{2}+\beta x-\gamma,
$$

with $\alpha=b_{1}+b_{2}+b_{3}, \beta=b_{1} b_{2}+b_{3} b_{1}, \gamma=b_{1} b_{2} b_{3}$. If we can find the symmetrical functions $\alpha, \beta, \gamma$, individual values for $b_{3}, b_{2}, b_{3}$ can be obtained from the cubic equation $x^{3}-\alpha x^{2}+\beta x-\gamma=0$. The essence of the factorisation method is to express $\beta$ and $\gamma$ as functions of $\alpha$ and then set up an equation which will give suitable values of $\alpha$. In the present problem, there can at most be eight factors of type $h(x)$ in $G(x)$, so we can expect $\alpha$ to satisfy an equation of degree 8. An additional consideration, because of equation (1.2), is that if $\left(b_{1}, b_{2}, b_{3}\right)$ provides a solution, then $\left(-b_{1},-b_{2},-b_{3}\right)$ is also a solution. In general, the two solutions will be distinct and $G(x)$ also has a factor $h^{*}(x)$, where

$$
h^{*}(x)=\left(x+b_{1}\right)\left(x+b_{2}\right)\left(x+b_{3}\right)=x^{3}+\alpha x^{2}+\beta x+\gamma \text {. }
$$


We can write

$$
\begin{aligned}
k(x) & =h(x) h^{*}(x)=\left(x^{3}+\beta x\right)^{2}-\left(\alpha x^{2}+\gamma\right)^{2} \\
& =x^{6}-A x^{4}+B x^{2}-C,
\end{aligned}
$$

where

$$
\begin{gathered}
A=b_{1}^{2}+b_{2}^{2}+b_{3}^{2}=\alpha^{2}-2 \beta, \quad C=b_{1}^{2} b_{2}^{2} b_{3}^{2}=\gamma^{2}, \\
B=b_{1}^{2} b_{2}^{2}+b_{2}^{2} b_{3}^{2}+b_{3}^{2} b_{1}^{2}=\beta^{2}-2 \alpha \gamma .
\end{gathered}
$$

At most there can be four factors of type $k(x)$ in $G(x)$ and the equation for $\alpha$ should reduce effectively to an equation of degree 4 . What happens in fact is that we get an equation of degree 4 in $\alpha^{2}$ and hence at most eight solutions for $\alpha$.

To relate $\beta$ and $\gamma$ to $\alpha$, we use the equations

$$
b_{i+1}=a b_{i}^{3}+(1-a) b_{1} \quad(i=1,2,3)
$$

with $b_{4}=b_{1}$ to ensure a cyclic solution. If we add the three equations

$$
\alpha=a \sum b_{\imath}^{3}+(1-a) \alpha,
$$

where the summation, here and subsequently, is from $i=1$ to $i=3$. Since $a$ is positive, this equation gives

$$
\alpha=\sum b_{l}^{3}=\alpha^{3}-3 \alpha \beta+3 \gamma
$$

or

$$
\gamma=(\alpha / 3)\left(1-\alpha^{2}+3 \beta\right) \text {. }
$$

With this equation established, we note that $\alpha=0$ now implies $\gamma=0$ and hence $b_{1}$ or $b_{2}$ or $b_{3}$ is zero. However if one of the $b_{1}$ is zero, equation (4.6) makes the others zero also and we are back to the equilibrium solution $x_{n}=0$. We deduce that $\alpha=0$ is inadmissible for a solution with minimum period 3. In the same way, no two of the $b_{t}$ can be equal without leading to an equilibrium solution. (Indeed, no two of the $b_{\imath}$ can be equal in magnitude for a solution with minimum period 3.)

From equation (4.6)

$$
b_{l} b_{t+1}=a b_{t}^{4}+(1-a) b_{i}^{2} \quad(i=1,2,3)
$$

and summing over $i$ gives

$$
\begin{aligned}
\beta & =a \sum b_{\imath}^{4}+(1-a) \sum b_{\imath}^{2} \\
& =a\left(\alpha^{4}-4 \alpha^{2} \beta+2 \beta^{2}+4 \alpha \gamma\right)+(1-a)\left(\alpha^{2}-2 \beta\right) .
\end{aligned}
$$

(From the theory of equations, any symmetrical function of $b_{1}, b_{2}, b_{3}$ can be expressed in terms of $\alpha, \beta$ and $\gamma$ and appropriate subsitutions have been made for $\Sigma b_{l}^{2}$ and $\Sigma b_{l}^{4}$ in equation (4.10). In the same way, $\Sigma b_{1}^{3}$ has been replaced by $\alpha^{3}-3 \alpha \beta+3 \gamma$ in equation (4.8). Similarly, we can write $\sum b_{l}^{4}=A^{2}-2 B$ and 
$\Sigma b_{i}^{6}=A^{3}-3 A B+3 C$.) If we substitute for $\gamma$ in equation (4.10), using equation (4.9), we obtain

$$
0=2 a \beta^{2}+(2 a-3) \beta+(1 / 3)\left\{(3+a) \alpha^{2}-a \alpha^{4}\right\} .
$$

In a sense, this gives $\beta$ as a function of $\alpha^{2}$ but in practice it proved better to re-write the equation as

$$
6 a \beta^{2}=(9-6 a) \beta+a \alpha^{4}-(3+a) \alpha^{2}
$$

and to use it to replace $\beta^{2}$ by a linear function of $\beta$ in other equations. For example,

$$
C=\gamma^{2}=\left(\alpha^{2} / 9\right)\left(1-\alpha^{2}+3 \beta\right)^{2}
$$

and using equation (4.12) leads to

$$
18 a C=\left\{2 a \alpha^{2}-(9+7 a) \alpha^{4}+5 a \alpha^{6}\right\}+\beta\left\{(27-6 a) \alpha^{2}-12 a \alpha^{4}\right\} .
$$

Similarly, the equation $B=\beta^{2}-2 \alpha \gamma$ leads to

$$
6 a B=\left(9-6 a-12 a \alpha^{2}\right) \beta+5 a \alpha^{4}-(3+5 a) \alpha^{2} .
$$

Since $A=\alpha^{2}-2 \beta$, these equations can easily be re-phrased in terms of $A$ and $\alpha^{2}$ rather than $\beta$ and $\alpha^{2}$.

We need two more equations, one to express $\beta$ in terms of $\alpha^{2}$ and another to eliminate $\beta$ and give an equation for $\alpha$. It turns out that there are several relationships available. From equation (4.6)

$$
\begin{aligned}
a^{2} \sum b_{t}^{6} & =\sum\left(a b_{l}^{3}\right)^{2}=\sum\left\{b_{i+1}-(1-a) b_{l}\right\}^{2} \\
& =A-2(1-a) \beta+(1-a)^{2} A
\end{aligned}
$$

and hence

$$
A\left(a^{2}-2 a+2\right)-2(1-a) \beta=a^{2}\left(3 C+A^{3}-3 A B\right) .
$$

After some reduction, using equations (4.13), (4.14), (4.4) and (4.12), we get

$$
\beta P_{1}(\alpha)=R_{1}(\alpha)
$$

where

$$
\begin{gathered}
P_{1}(\alpha)=9+\left(6 a-10 a^{2}\right) \alpha^{2}+10 a^{2} \alpha^{4}, \\
R_{1}(\alpha)=\alpha^{2}\left\{\left(3-9 a+6 a^{2}\right)+\left(3 a-10 a^{2}\right) \alpha^{2}+4 a^{2} \alpha^{4}\right\} .
\end{gathered}
$$

For a given value of $a$, this gives $\beta$ as a rational function of $\alpha^{2}$.

Another relationship can be obtained by multiplying together the three equations (4.6) to give

$$
b_{2} b_{3} b_{1}=\left(b_{1} b_{2} b_{3}\right) \prod_{i=1}^{3}\left\{a b_{i}^{2}+(1-a)\right\}
$$


Since none of the $b_{t}$ can be zero, we can cancel $b_{1} b_{2} b_{3}$ from both sides and obtain

$$
1=a^{3} C+a^{2}(1-a) B+a(1-a)^{2} A+(1-a)^{3} \text {. }
$$

Substituting for $A, B$ and $C$ in terms of $\alpha^{2}$ and $\beta$ leads to

$$
\beta P_{2}(\alpha)=R_{2}(\alpha) \text {, }
$$

where

$$
\begin{aligned}
P_{2}(\alpha)=9 & -27 a+18 a^{2}+\left(9 a-30 a^{2}\right) \alpha^{2}+12 a^{2} \alpha^{4}, \\
R_{2}(\alpha)= & -18\left(a^{2}-3 a+3\right)+\left(9-42 a+35 a^{2}\right) \alpha^{2} \\
& +\left(6 a-22 a^{2}\right) \alpha^{4}+5 a^{2} \alpha^{6} .
\end{aligned}
$$

Yet another relationship arises from equation (4.6) if we note that

$$
b_{3}-b_{2}=\left\{b_{2}-b_{1}\right\}\left\{a\left(b_{1}^{2}+b_{1} b_{2}+b_{2}^{2}\right)+(1-a)\right\} .
$$

There will be two similar equations for $b_{1}-b_{3}$ and $b_{2}-b_{1}$ and we can multiply the three equations together, cancel a non-zero factor $\left(b_{3}-b_{2}\right)\left(b_{1}-b_{3}\right)\left(b_{2}-b_{1}\right)$ on either side and get a symmetric equation

$$
\begin{aligned}
1= & \prod_{i=1}^{3}\left\{a\left(b_{i}^{2}+b_{i} b_{i+1}+b_{i+1}^{2}\right)+(1-a)\right\} \\
= & a^{3}(A B+B \beta+A \alpha \gamma)+a^{2}(1-a)\left(\alpha^{2}+3 \alpha \gamma+3 B\right) \\
& +a(1-a)^{2}(2 A+\beta)+(1-a)^{3} .
\end{aligned}
$$

This equation is similar to equation (4.20) but messier to reduce. However it leads to

$$
\beta P_{3}(\alpha)=R_{3}(\alpha)
$$

where

$$
\begin{aligned}
& P_{3}(\alpha)=\left(9-18 a+12 a^{2}\right)+\left(12 a-26 a^{2}\right) \alpha^{2}+14 a^{2} \alpha^{4} \\
& R_{3}(\alpha)=-12\left(a^{2}-3 a+3\right)+\left(15-39 a+28 a^{2}\right) \alpha^{2} \\
&+\left(9 a-22 a^{2}\right) \alpha^{4}+6 a^{2} \alpha^{6}
\end{aligned}
$$

(In much the same way, we can use

$$
b_{3}+b_{2}=\left(b_{2}+b_{1}\right)\left\{a\left(b_{1}^{2}-b_{1} b_{2}+b_{2}^{2}\right)+(1-a)\right\}
$$

and the two similar equations to generate an equation for $\beta$ but we already have enough equations for our purpose.)

We can obtain the desired equation for $\alpha^{2}$ by eliminating $\beta$ between any two of the equations (4.17), (4.21) and (4.26) or, if we wish to save a little bit of algebra, we can use a linear combination of all three to give a simpler equation. If we take $3 P_{3}(\alpha)-2 P_{2}(\alpha)-P_{1}(\alpha)$ we get $4 \alpha^{2}$ as a factor and the same factor occurs in 
$3 R_{3}(\alpha)-2 R_{2}(\alpha)-R_{1}(\alpha)$. If we write

$$
\begin{aligned}
& P_{4}(\alpha)=3 P_{3}(\alpha)-2 P_{2}(\alpha)-P_{1}(\alpha)=4 \alpha^{2} P_{5}(\alpha), \\
& R_{4}(\alpha)=3 R_{3}(\alpha)-2 R_{2}(\alpha)-R_{1}(\alpha)=4 \alpha^{2} R_{5}(\alpha),
\end{aligned}
$$

then

$$
\beta P_{5}(\alpha)=R_{5}(\alpha)
$$

with

$$
\begin{aligned}
P_{5}(\alpha) & =\left(3 a-2 a^{2}\right)+2 a^{2} \alpha^{2}, \\
R_{5}(\alpha) & =\left(6-6 a+2 a^{2}\right)+\left(3 a-3 a^{2}\right) \alpha^{2}+a^{2} \alpha^{4} .
\end{aligned}
$$

(It was noted earlier that $\alpha$ cannot be zero for a solution with minimum period 3.)

Eliminating $\beta$ between equations (4.17) and (4.29) gives $P_{1}(\alpha) R_{5}(\alpha)=$ $P_{5}(\alpha) R_{1}(\alpha)$ as the equation for $\alpha$. This condition leads to an equation

$$
N(X, a)=0 \text {, }
$$

where $X=\alpha^{2}$ and

$$
\begin{aligned}
N(X, a)= & \left(27-27 a+9 a^{2}\right)+\left(27 a-45 a^{2}+18 a^{3}-4 a^{4}\right) X \\
& +\left(36 a^{2}-27 a^{3}+9 a^{4}\right) X^{2}+\left(9 a^{3}-6 a^{4}\right) X^{3}+a^{4} X^{4} .
\end{aligned}
$$

\section{Critical values and stability intervals}

We now have enough equations to go over to computational work. For a given value of the parameter $a$, equation (4.32) becomes a fourth degree equation in $X$ and we can use numerical methods to find the positive roots, if any, of this equation. If $X_{0}$ is a positive root, we can take $\alpha=\sqrt{X_{0}}$ and calculate $\beta$ from equation (4.29), say, and $\gamma$ from equation (4.9). The roots of the equation

$$
0=h(x)=x^{3}-\alpha x^{2}+\beta x-\gamma
$$

now give $\left(b_{1}, b_{2}, b_{3}\right)$ for the corresponding solution of period 3. As noted before, $\left(-b_{1},-b_{2},-b_{3}\right)$ also gives a solution and this is the solution obtained by taking $\alpha=-\sqrt{X_{0}}$ instead of $\alpha=+\sqrt{X_{0}}$. In the subsequent discussion, we shall consider only solutions with $\alpha>0$ and at most there will be four of these solutions. The mirror image solutions still exist, of course, but we can ignore them in enumerating the solutions for a given value of $a$. With this convention, we can summarise the results by saying that there are two solutions for $3.70<a<3.925$ and four solutions for $3.925<a \leqslant 4$. Thus there are two critical values for $a$ and for each critical value there is a value of $X$ at which $N=0$ and $\partial N / \partial X=0$. The graph of $N$ against $X$ has a minimum which touches the $X$-axis at this critical value of $X$ and an increase in $a$ gives two separate zeros for $N(X, a)$. 
The condition for stability is that $-1<S_{3}<1$, where

$$
\begin{aligned}
S_{3} & =F^{\prime}\left(b_{3}\right) F^{\prime}\left(b_{2}\right) F^{\prime}\left(b_{1}\right)=\prod_{i=1}^{3}\left\{3 a b_{\imath}^{2}+(1-a)\right\} \\
& =27 a^{3} C+9 a^{2}(1-a) B+3 a(1-a)^{2} A+(1-a)^{3} .
\end{aligned}
$$

For the critical values of $a$ and $X, S_{3}=+1$ and the solution is at the limiting value for stability. As $a$ increases beyond the critical value, two distinct solutions occur, with $S_{3}>1$ for one solution and $S_{3}<1$ for the other. We can think of these as separate families of solutions, with instability for all the solutions in one case and with a small interval of stability in the other case before $S_{3}$ becomes less than -1. (Because $S_{3}$ is a function of $b_{i}^{2}$, the value of $S_{3}$ is the same for a pair of mirror image solutions and they are both stable if one of them is stable.)

Some guidance about the stability can be obtained from the solutions for $a=4$. For $a=4$, equation (5.2) gives

$$
S_{3}=27(64 C-16 B+4 A-1)
$$

and exact expressions for $A, B, C$ are available in this case. To indicate how they arise, note that

$$
\begin{aligned}
N(X, 4) & =256 X^{4}-960 X^{3}+1152 X^{2}-484 X+63 \\
& =\left(16 X^{2}-32 X+7\right)\left(16 X^{2}-28 X+9\right)
\end{aligned}
$$

and the roots of $N(X, 4)=0$ can be written down, in order of increasing magnitude, as

$$
X_{1}=1 / 4, \quad X_{2}=(7-\sqrt{13}) / 8, \quad X_{3}=(7+\sqrt{13}) / 8, \quad X_{4}=7 / 4 .
$$

These values are shown in Table 1 , with the corresponding values of $\alpha, \beta, \gamma, A, B, C, S_{3}$ and $\phi$. The numerical calculations indicate that $X_{1}$ and $X_{2}$ correspond to the two solutions which first appear around $a=3.70$ and Table 1 shows that the solution with the smaller $X$ value is the one to use in looking for a stability interval. In the same way, $X_{3}$ and $X_{4}$ correspond to the two solutions which first appear around $a=3.925$ and the solution with the larger $X$ value provides a stability interval in this case.

TABLE I. Values of $X, \alpha, \beta, \gamma, A, B, C, S_{3}, \phi$ for $a=4$. (In this table, $q$ denotes $\sqrt{13}$.)

\begin{tabular}{|c||c|c|c|c|}
\hline & Solution 1 & Solution 2 & Solution 3 & Solution 4 \\
\hline$X$ & $1 / 4$ & $(7-q) / 8$ & $(7+q) / 8$ & $7 / 4$ \\
$\alpha$ & $1 / 2$ & $(-1+q) / 4$ & $(1+q) / 4$ & $\sqrt{7} / 2$ \\
$\beta$ & $-1 / 2$ & $-1 / 4$ & $-1 / 4$ & 0 \\
$\gamma$ & $-1 / 8$ & $(3-q) / 16$ & $-(3+q) / 16$ & $-\sqrt{7} / 8$ \\
$A$ & $5 / 4$ & $(11-q) / 8$ & $(11+q) / 8$ & $7 / 4$ \\
$B$ & $3 / 8$ & $(9-2 q) / 16$ & $(9+2 q) / 16$ & $7 / 8$ \\
$C$ & $1 / 64$ & $(11-3 q) / 128$ & $(11+3 q) / 128$ & $7 / 64$ \\
$S_{3}$ & -27 & +27 & +27 & -27 \\
$\phi$ & $2 \pi / 14$ & $2 \pi / 13$ & $\pi / 13$ & $\pi / 14$ \\
\hline
\end{tabular}


The end results of the calculations are shown in Table 2. In the table, $X_{1}, X_{2}, X_{3}$ and $X_{4}$ denote the roots of $N(X, a)=0$ in ascending order of magnitude, while $a^{*}$ is the critical value of $a$ and $X^{*}$ the critical value of $X$ at which these roots first appear. The next column shows which roots gives an interval of stable solutions. The upper limit of $a$ for stable solutions is denoted by $a^{* *}$ and appears below $a^{*}$ in column 2. The cyclic solution for $a=a^{*}$ (with $\alpha>0$ ) is also included as an indication of the type of solution obtained. It is easy to see from the corresponding directed graph [6] that once a solution of period 3 appears, a solution with any period is possible. However, the narrow intervals of stability and the extent of the frequency changes involved suggest that solutions of period 3 are unlikely to occur in practice in genetic problems.

TABLE 2. Critical values for solutions of period 3.

\begin{tabular}{|c|c|c|c|c|}
\hline$X$-values & $\begin{array}{c}a^{*} \\
a^{* *}\end{array}$ & $X^{*}$ & $\begin{array}{c}\text { Stable } \\
\text { sequence }\end{array}$ & $\begin{array}{c}\text { Typical solution } \\
\left(b_{1}, b_{2}, b_{3}\right)\end{array}$ \\
\hline$X_{1}, X_{2}$ & 3.69964 & 0.34855 & $X_{1}$ & $0.8874,0.1896,-0.4866$ \\
& 3.70232 & & & \\
\hline$X_{3}, X_{4}$ & 3.92487 & 1.51397 & $X_{4}$ & $0.9718,0.7597,-0.5011$ \\
\hline
\end{tabular}

To complete the discussion, two minor points should be mentioned. One is that an equation such as (4.17) can easily be changed to an equation for $A$, since $2 \beta=\alpha^{2}-A$. Thus we can write

$$
A P_{1}(\alpha)=\alpha^{2} P_{1}(\alpha)-2 R_{1}(\alpha)=Q_{1}(\alpha),
$$

with similar equations for $A P_{2}(\alpha), A P_{3}(\alpha)$ and $A P_{5}(\alpha)$. In the actual calculations, these equations were used instead of the equations for $\beta$ as the starting point in obtaining $A, B, C, \beta, \gamma$. The values for $A$ must be positive and in the examples considered they were of order unity, whereas $\beta$ could have either sign and in some instances $|\beta|$ was small. (Table 1 includes a solution where $\beta=0$.)

The second point is that $N(X, a)$ has an isolated zero for $X=0.4$ and $a=2.5$, a zero which does not give a starting-point for admissible families of solutions. What happens is that $N$ has a minimum at $(0.4,2.5)$ when regarded as a function of two variables, so $N$ is positive for all neighbouring values of $X$ and $a$ and we do not get solutions of $N(X, a)=0$ when $a$ is increased slightly. At the point $(0.4,2.5)$, equations (4.17), (4.21), (4.26) and (4.29) give indeterminate values for $\beta$ but equation (4.11) becomes

$$
0=5 \beta^{2}+2 \beta+3 / 5
$$

and this gives complex values for $\beta$. Thus the zero of $N(X, a)$ at $(0.4,2.5)$ does not correspond to a real solution. 


\section{Acknowledgements}

I should like to thank Professor R. M. May for sending me a copy of his lecture notes for the Les Houches Summer School [9]. The work has been done while appointed as a Visiting Fellow in the Research School of Physical Sciences, Australian National University, and I am grateful to the Department of Theoretical Physics for the facilities provided.

\section{References}

[1] A. Brown, "Equations for periodic solutions of a logistic difference equation", J. Austral. Math. Soc. Ser. B 23 (1981), 78-94.

[2] A. Brown, "Solutions of period seven for a logistic difference equation", Bull. Austral. Math. Soc. 26 (1982), 263-284.

[3] B. C. Clarke, "Frequency-dependent and density-dependent natural selection", in The role of natural selection in human evolution (ed. F. M. Salzano), (North-Holland, Amsterdam, 1975), $187-200$.

[4] B. C. Clarke, "The evolution of genetic diversity", Proc. Roy. Soc. London Ser. B 205 (1979), 453-474.

[5] D. S. Falconer, Introduction to quantitative genetıcs (Longman, London, 2nd edition, 1981), 43.

[6] J. C. Frauenthal, Introduction to population modeling (Birkhäuser, Basel, 1980), 71-73.

[7] R. C. Lewontin, The genetıc basis of evolutınary change (Columbia Univ. Press, New York, 1974), 256-260.

[8] R. M. May, "Bifurcations and dynamic complexity in ecological systems", Ann. N. Y. Acad. Scl. 316 (1979), 517-529.

[9] R. M. May, "Non-linear problems in ecology and resource management", Lecture notes for Les Houches Summer School on "Chaotic Behaviour of Deterministic Systems", July 1981.

[10] D. T. Parkin, An introduction to evolutionary genetics (Arnold, London, 1979), 81, 99-110. 